\section{Literatur}

1. Singh RP, Agarwal R (2006) Prostate cancer chemoprevention by silibinin: bench to bedside. Mol Carcinog 45: 436-442

2. Setchell KD, Cassidy A (1999) Dietary isoflavones: biological effects and relevance to human health. J Nutr 129: 758S-767S

3. Surh YJ (2003) Cancer chemoprevention with dietary phytochemicals. Nat Rev Cancer 3: 768-780

4. Sonn GA, Aronson W, Litwin MS (2005) Impact of diet on prostate cancer: a review. Prostate Cancer Prostatic Dis 8: 304-310

5. Nelson PS, Montgomery B (2003) Unconventional therapy for prostate cancer: good, bad or questionable? Nat Rev Cancer 3: 845-858

6. Taplin ME (2007) Drug insight: role of the androgen receptor in the development and progression of prostate cancer. Nat Clin Pract Oncol 4: 236-244

7. Hellawell GO, Turner GD, Davies DR et al. (2002) Expression of the type 1 insulin-like growth factor receptor is up-regulated in primary prostate cancer and commonly persists in metastatic disease. Cancer Res 62: 2942-2950

8. Chen CD, Welsbie DS, Tran C et al. (2004) Molecular determinants of resistance to antiandrogen therapy. Nat Med 10: 33-39

9. Kuiper GG, Lemmen JG, Carlsson B et al. (1998) Interaction of estrogenic chemicals and phytoestrogens with estrogen receptor beta. Endocrinology 139: 4252-4263

Urologe 2007 • 46:1274-1278 • DOI 10.1007/s00120-007-1497-0 • Online publiziert: 1. August 2007

(c) Springer Medizin Verlag 2007

\title{
G. Thalmann
}

Urologische Universitätsklinik, Anna Seiler-Haus, Bern

\section{Projekte des 6. EU-Rahmenprogramms}

\section{PROMET}

Im April 2006 hat das auf vier Jahre angelegte Projekt PROMET („PROstate cancer Molecular-oriented detEction and Treatment of minimal residual disease") begonnen. Sein Ziel ist die Detektion von Mikrometastasen (minimale Resterkrankung, „minimal residual disease“) und die Entwicklung neuer Therapiestrategien für diese. Dieses Forschungsprojekt mit spezifischen Zielen („specific targeted research project“, STREP) wird finanziert vom 6. EU-Rahmenprogramm (Infobox 1).

In der EU wird jährlich bei etwa 200.00o Männern ein Prostatakarzinom diagnostiziert, und angesichts der durch Alterung wachsenden Risikopopulation wird diese Zahl in Zukunft wohl noch steigen. Dank der Fortschritte in der Behandlung des Primärtumors hängt die Mortalität von Krebspatienten immer mehr mit Metastasen zusammen, die zum Zeitpunkt der Diagnose bzw. Therapie des Primärtumors oft okkult sind (Mikrometastasen, „minimal residual disease“). Nach radikaler Therapie mit kurativer Zielsetzung bei Patienten mit lokal begrenzter Erkrankung kommt es bei 20$30 \% \mathrm{zu}$ einem Wiederauftreten und schließlich zur Metastasenbildung.

Maligne Tumoren sind bekanntermaßen heterogen, und die biologischen Eigenschaften von Subpopulationen mit unterschiedlichem invasiven und metastatischen Potenzial können sich ändern: im Lauf der Zeit und unter der Behandlung, u. a. aufgrund von genetischer Instabilität und epigenetischen Einflüssen. Der Primärtumor gibt eine große Anzahl von Zellen in den Blutkreislauf ab, doch nur eine ganz kleine Minderheit (etwa o,01\%) dieser Tumorzellen wird letztlich in der
Lage sein, metastatische Absiedlungen zu bilden.

Für die frühzeitige Erkennung einer minimalen Resterkrankung ist es in Zukunft unerlässlich, die komplexen Mechanismen, über die sich aus zirkulierenden Tumorzellen Mikrometastasen und Metastasen entwickeln, auf molekularer und physiologischer Ebenen zu verstehen. Die Fähigkeit, Mikrometastasen mit dem Potenzial der Metastasenbildung zu erkennen, wird es ermöglichen, Voraussagen zum Outcome zu treffen und letztendlich neue Behandlungsmodalitäten zu entwickeln.

Daher schlagen wir vor, im Rahmen dieses STREP die Mechanismen und die genetische Signatur der minimalen Resterkrankung (MRD) beim Prostatakarzinom zu ergründen und neuartige therapeutische Ansätze zu entwickeln, um das

Infobox 1 PROMET - Institute teilneh-
mender Partner
- Departement für Urologie, Universität
Bern, Schweiz (Koordinierung)
- Institut für Angewandte Physik,
Universität Bern, Schweiz
- Departments of Urology and Endocrino-
logy, Leiden University Medical Center,
Niederlande
- Department of Urology, University of
Sheffield, Sheffield, England
- INSERM, University of Lyon, Lyon,
Frankreich
- Deutsches Krebsforschungszentrum,
Heidelberg, Deutschland
- Kontron Medical S.A.S., Basel, Schweiz
- Berthold Technologies GmbH \& Co. KG,
Bad Wildbad, Deutschland
- Apoxis S.A., Lausanne, Schweiz
- tp21 GmbH, Saarbrücken, Deutschland
- Med Discovery S.A., Lausanne, Schweiz
http://www.fp6-promet.net.


Wachsen einer MRD zu einer Metastase zu verhindern. In enger Zusammenarbeit klinischer Forscher mit Grundlagenforschern werden die Mechanismen der MRD untersucht. Dabei kommen u. a. zum Einsatz: funktionelle Genomik und Expressionsprofiling, fortgeschrittene experimentelle MRD-Modelle sowie Biolumineszenz- und Multiphotonenmikroskopie. Potenzielle neue Behandlungsstrategien und sensitivere bildgebende Modalitäten (entwickelt von der Industrie und ausgewählt wegen ihres Potenzials zur Früherkennung und Eradikation einer MRD, beispielsweise $\mathrm{Na}$ no- und optoakustische Technologien) werden an experimentellen präklinischen Modellen für die nachfolgende klinische Evaluierung getestet.

Ziel des Projekts ist die Entwicklung eines diagnostischen Tests für die Detektion einer minimalen Resterkrankung und für die Identifizierung von mindestens zwei Signaltransduktionstargets für neuartige Strategien zur Behandlung von Mikrometastasen. Zu den weiteren therapeutischen Ansätzen gehört die Evaluierung synthetischer Fusionsproteine, die von Apoxis (Lausanne, Schweiz) mit Hilfe ihrer patentrechtlich geschützten Multimerisierungstechnologie für die Apoptoseinduktion über den Fas-Pfad hergestellt werden, sowie ein rekombinanter, für humanes Kallikrein 2 spezifischer Inhibitor, der von Med Discovery (Lausanne, Schweiz) unter Verwendung von über Phagen-Displays ausgewählten Substraten entwickelt wurde.

Die Ergebnisse werden in Publikationen mit Peer Review veröffentlicht. Mögliche klinische und präklinische Anwendungen können zur Patentierung angemeldet werden.

\section{PRIMA („PRostate Cancer Integral Management Approach", Prosta- takrebs: ganzheitlicher Managementansatz)}

PRIMA ist ein ganzheitliches Projekt („integrated project“, IP), das von Prof. Jack Schalken, Nijmegen, Niederlande, koordiniert und von der EU-Kommission im Rahmen des EU-Forschungsrahmenprogramms teilfinanziert wird. Das Departement für Urologie der Universität Bern nimmt teil an diesem Projekt, das im Juli 2004 begonnen wurde (Infobox 2).

Für das lokal begrenzte Prostatakarzinom sind zwar radikale Therapien verfügbar, die auf die Eradikation des Karzinoms zielen und den Patienten heilen können, doch gibt es derzeit keine effektive Therapie für das lokal fortgeschrittene und das metastasierte Prostatakarzinom. Die Androgendeprivation hat in den meisten Fällen nur einen zeitlich begrenzten Effekt, ist also vor allem eine palliative Therapie. Zahlreiche therapeutische Strategien, die auf ganz unterschiedliche Zielstrukturen gerichtet sind, befinden sich zurzeit in präklinischen und/oder klinischen PhaseI-Studien. Bisher haben sich die meisten „targeted approaches“ als nicht erfolgreich erwiesen.

Für dieses Projekt werden multidisziplinäre Bemühungen vorgeschlagen, um die tödlichste Art von Prostatakrebs - das hormonrefraktäre, ossär metastasierte Prostatakarzinom - auf verschiedenen Ebenen zu bekämpfen. Dabei werden wir neue Komponenten unter Verwendung maßgeschneiderter Hochdurchsatz-Screeningassays auswählen, und so werden sich wahrscheinlich weniger naheliegende Präparate identifizieren lassen und patentierbare Zielstrukturen identifizieren lassen.

Durch die Integration unterschiedlicher State-of-the-art-Ansätze wollen wir mindestens fünf molekulare Ziele identifizieren und validieren, außerdem Präparate, die in Tiermodellen für ossär metastasierten Prostatakrebs präklinisch getestet werden können.

Die verschiedenen Interventionsebenen beginnen mit dem Androgenrezeptor (AR), also auf der Ebene der ARProtein-Interaktion, der nichtsteroidalen Aktivierung des AR, der nichttranskriptionalen Funktion des AR, der Expression und Struktur von Kofaktoren, funktionalen Studien von AR-Koaktivatoren und schließlich mit dem Angriff auf AR-Response-Gene.

Die nächste Herangehensebene besteht im Expressionsprofiling der TGF $\beta$-Superfamilie, einer Familie von Genen, die wesentlich an der Karzinogenese von Prostatakrebs beteiligt sind. Untersucht werden weiterhin die Interaktionen zwischen TGF $\beta$, BMPs und ihren jeweiligen Anta-
Infobox 2 PRIMA

Teilnehmende Institutionen

- Department of Experimental Urology, University Medical Centre Nijmegen (Coordination), Niederlande

- Departments of Pathology \& Urology, Erasmus Medical Centre Rotterdam, Niederlande

- Molecular Pathology / Structural Biology \& Genomics, INSERM/IGBMC, Straßburg, Frankreich

- Department of Urology, University of Sheffield, England

- Medical Biotechnology Department, University of Turku, Finnland

- Compound Handling B.V., Delft, Niederlande

- Department of Urology, Innsbruck Medical University, Österreich

- Department of Urology, University of Newcastle, England

- Institute of Toxicology and Genetics, Forschungszentrum Karlsruhe, Deutschland

- Department of Urology, University of Bern, Schweiz

- Department of Biology, University of York, USA

- Centre de Recherche pour les Pathologies Prostatiques, Paris, Frankreich

- Departments of Endocrinology \& Urology, Leiden University Medical Centre, Niederlande

- Institute of Medical Technology, University of Tampere, Finnland

- Department of Molecular Cell Biology, Weizmann Institute of Science, Rehovot, Israel

- Departments of Surgery \& Oncology, University of Cambridge, England

Infobox 3 PRIMA - Publikationen

- Sapountzi V, IR Logan, CN Robson (2006) Cellular functions of TIP60. Int J Biochem Cell Biol 38: 1496-1509

- Kogan I, Goldfinger N, Milyavsky M, Cohen M, Shats I, Dobler G, Klocker H, Wasylyk B, Voller M, Aalders T, Schalken JA, Oren M, Rotter V (2006) hTERT-Immortalized prostate epithelial and stromal-derived cells: an authentic in vitro model for differentiation and carcinogenesis. Cancer Res 66: 3531-3540

- Rentsch CA, Cecchini MG, Schwaninger R, Germann M, Markwalder R, Heller M, van der Pluijm G, Thalmann GN, Wetterwald A (2006) Differential expression of TGFb-stimulated clone 22 in normal prostate and prostate cancer. Int J Cancer 118: 899-906

- Schwaninger R, Rentsch CA, Wetterwald A, van der Horst $G$, van Bezooijen RL, van der Pluijm G, Löwik CWGM, Ackermann K, Pyerin W, Hamdy FC, Thalmann GN, Cecchini MG (2007) Lack of noggin expression by cancer cells is a determinant of the osteoblast response in bone metastases. Am J Pathol 170: 160-175 
gonisten sowie die Rolle von Osteoprotegerin (OPG) bei der ossären Metastasierung. Es gibt vermehrt Hinweise darauf, dass der physiologische Prozess des epithelialen-mesenchymalen Übergangs („epithelial-mesenchymal transition“, EMT), der während der Embryonalentwicklung eine wichtige Rolle spielt, wesentlich für den Prozess der Metastasierung ist. TGF $\beta$ ist beteiligt an EMT, und das Konsortium ist dabei, ein In-vitroModell für EMT zu entwickeln. Darüber hinaus werden neue, stabile, immortalisierte Prostatakrebs-Zelllinien etabliert.

Neuartige Targets und genomische Instrumente werden vom Konsortium evaluiert und dann für das präklinische Screening von in Frage kommenden Präparaten verwendet. Mit Hilfe der PRIMA-Bioinfomatikplattform in Straßburg wird das Konsortium neue Kandidaten-Targets isolieren, die dann mit Methoden der funktionellen Genomik sowie an experimentellen Modellen und in Humangeweben (Infobox 3) evaluiert werden.

\section{CANCURE - Trainee-Programm für die Erforschung früher Krebsstadien}

Es besteht dringender Bedarf, Wissenschaftler für optimale Forschung mit multidisziplinären und integrierten Ansätzen auszubilden, wenn sie Teams zusammenstellen und Projekte entwickeln sollen, die den sich aus den Fortschritten in Systembiologie, Bildgebung, funktioneller Analyse, Hochdurchsatzscreening und Bioinformatik ergebenden Herausforderungen besser gewachsen sind.

CANCURE ist ein paneuropäisches Trainee-Programm, das vom 6. EU-Forschungsrahmenprogramm finanziert wird. Das Forschungstraining ist konzipiert für den Bereich der translationalen Krebsforschung mit dem Prostatakarzinom als Modellsystem. Dabei werden hochentwickelte molekular- und zellbiologische experimentelle Techniken sowohl in vitro als auch in vivo an biologischen Modellsystemen angewendet. Die Ausbildung orientiert sich an den Bedürfnissen aller im Bereich der frühen Tumorentstehung Forschenden („early stage researchers", ESR). Auch wenn die translationale Erforschung des Prostatakarzinoms im Mittelpunkt des Programms steht, so werden die beteiligten Forscher doch auch multidisziplinäre Kompetenzen und Rüstzeug erwerben, die sie in anderen mit der Humanpathologie zusammenhängenden Gebieten nutzen können.

CANCURE ist ein prestigeträchtiges vierjähriges Trainee-Programm für Doktoranden, finanziert von der EU-Kommission, die elf führende Forschergruppen aus sieben europäischen Ländern in diesem multidisziplinären translationalen Krebsforschungsprojekt zusammenführt, das sich auf Prostatakrebs konzentriert. Die gut dotierten Stellen bieten den Absolventen eine einzigartige Möglichkeit, auf dem Gebiet der Krebsforschung ein breites Fundament an Kompetenzen zu erwerben. Die Koordination liegt in den Händen von Craig Robson, Professor für Molekulare Urologie in Newcastle, England.

Die Laborausbildung der einzelnen Forschenden wird zwar in erster Linie im Labor des Gastinstitutes stattfinden, doch werden die meisten Doktoranden auch einige Zeit im Labor eines weiteren CANCURE-Partners verbringen, um zusätzliche für ihr Forschungsgebiet relevante Fähigkeiten zu erwerben. Die im Bereich der frühen Tumorentstehung Forschenden erhalten Anleitung und Monitoring von einem Beraterkomitee, das aus Kapazitäten auf dem Gebiet der translationalen Forschung, erfahrenen Universitätsprofessoren, externen Experten sowie lokalen Mentoren besteht. Die Trainees werden an jährlich stattfindenden Sommerworkshops und an CANCURE-spezifischen Seminaren teilnehmen, an internationalen Seminaren und Kursen zu Themen wie interpersonelle Kompetenzen, Fremdsprachen und Ethik. Um auf europäische Doktorgrade hinzuarbeiten, werden optimale Praktiken („best practices“) eingeübt, wie sie beispielsweise in den Statuten des internationalen EMBL (European Molecular Biology Laboratory)-PhD-Programms empfohlen werden. Die Teilnehmenden sollen befähigt werden, zu Labors in Wissenschaft oder Industrie zurückzukehren, dort integrierte, multidisziplinäre Forschungsprojekte zu entwickeln und entsprechende Teams zusammenzustellen, modernste Technologien anzuwenden, auf der im Kurs etablierten Zusam- menarbeit aufzubauen und so die Wettbewerbsfähigkeit Europas zu verbessern.

Das CANCURE-Forschungsprogramm stützt sich sowohl auf beendete als auch auf laufende EU-finanzierte Kooperationen zwischen den elf beteiligten Partnern. Für die elf Forscher im Bereich der frühen Stadien haben die CANCUREPartner drei schwerpunktmäßig zu bearbeitende Bereiche festgelegt:

- Umgehung der Androgenrezeptor (AR)-Regulierung bei hormonrefraktärem Prostatakrebs,

- Veränderungen der Eigenschaften von Prostatakarzinomzellen vor der ossären Metastasierung und

- Beitrag noch nicht entdeckter Mechanismen zum Progress von Prostatakrebs.

Schematisch gibt es vier Bereiche, die jeweils unterschiedliche fachliche Kompetenzen erfordern:

1. Phänotypische Analyse - An Modellsystemen entdeckte biologische Prozesse sind möglicherweise nicht relevant für Menschen und die menschliche Prostata. Zugang zu ganz klar - auch molekularbiologisch - charakterisierten Tumoren und fachkundige Interpretation der Daten kann nur in spezialisierten Zentren erfolgen. Die Projekte erfordern die phänotypische Analyse von bioptisch gewonnenem Material der Patienten.

2. Funktionale Analyse - Ein zweiter grundlegender Schritt ist die Verwendung von State-of-the-art-Systembiologie für die Analyse von an Prostatakarzinomen beteiligten Molekülen. Diese funktionellen wie bioinformatischen Analysen werden von der Bündelung von Expertise in den spezialisierten Exzellenzzentren profitieren.

3. Modellsysteme - Prostatakrebs wird an zahlreichen Modellsystemen untersucht, sowohl an spezialisierten (ProstataZelllinien, Xenografts, spezifische transgene Maus-Zelllinien) als auch an generischen Systemen (Mäuse, Fische, Würmer und Fliegen).

4. Translation - Forschungsergebnisse werden auf unterschiedlichen Wegen in die Praxis übertragen, so auch durch Entwicklung von Medikamenten mithilfe von Datenbankscreenings, Identifikation von Markern in Körperflüssigkeiten, Wieder- 
einleitung der Moleküle auf dem Wege der Gentherapie und auf klinischen Wegen.

Für das CANCURE-Programm sind elf Doktoranden in elf Forschungsinstitutionen in sieben EU-Ländern rekrutiert worden. Gastgebende Institutionen sind Newcastle University, England; Centre Européen de Recherche en Biologie et en Médicine, Frankreich; Forschungszentrum Karlsruhe $\mathrm{GmbH}$, Deutschland; Universität Bern, Schweiz; Cambridge University, England; Radboud University Nijmegen Medical Centre, Niederlande; York University, England; Universität Innsbruck, Österreich; Sheffield University, England; University of Tampere, Finnland; Erasmus Medical Centre, Rotterdam, Niederlande.

\section{Europäische Labors für moleku- lare Bildgebung (EMIL)}

EMIL („European Molecular Imaging Laboratories") ist ein Exzellenznetzwerk, das europäische Labors für molekulare Bildgebung versammelt mit dem gemeinsamen Ziel der Krebsbekämpfung. Arbeitsgruppen mit Projekten und Fachkenntnis in den Bereichen bildgebende Techniken, bildgebende Instrumente, neue Tiermodelle, molekulare Targets zur Darstellung von malignem Wachstum und Apoptose, Bildgebung und Follow-up von gesteuerten Therapien sowie Medikamentenentwicklung werden hier zusammengebracht. EMIL wird finanziert von der EU-Kommission mit der inhaltlichen Priorität Life Sciences für Gesundheit/Krebsbekämpfung. Das Programm (Projekt-Nummer: LSHC-CT-2004-503569) wurde am 1. Juli 2004 für eine Dauer von fünf Jahren begonnen. EMIL koordiniert Forschungsbemühungen auf dem Gebiet der molekularen Bildgebung von Krebserkrankungen in Europa mit 59 Gruppen aus Universitäten, Forschungszentren und der Industrie und soll die gemeinsame Nutzung von Fachwissen, Know-how und anderen intellektuellen Ressourcen erleichtern.

- EMIL verfügt über sechs Podien für

Technologien, Training und Fortbil-

dung, wissenschaftlichen Austausch,

industrielle Partnerschaften und Mobilität der Forschenden.

- EMIL fördert die theoretische und experimentelle Fortbildung sowie offene und spezialisierte Workshops auf hohem Niveau.

Das EMIL Forschungsprogramm richtet sich auf sieben Hauptziele:

1. Optimierung von Hard- und Softwaretechniken für die Integration von Daten aus Radiotracer-, Magnetresonanz- und optischen Bildgebungsverfahren. Dies erfordert die Entwicklung spezieller Instrumente und ihre Validierung für die Krebsforschung sowie von Software für die gemeinsame Registrierung, die Quantifizierung und die Weiterverarbeitung multimodaler Daten.

2. Entwicklung sog. ,intelligenter“ Bildgebungsinstrumente, die für einzelne molekulare Vorgänge spezifisch sind und die von mindestens einer bildgebenden Modalität erkannt und lokalisiert werden können.

3. Verwendung und Weiterentwicklung von Mausmodellen für maligne Tumoren beim Menschen, um die Früherkennung kleiner Tumoren durch hochentwickelte Bildgebung zu verbessern und um über einen längeren Zeitraum in demselben Tier Veränderungen in der Expression von Genen sowie Tumorzellproliferation und -migration in vivo zu untersuchen.

4. Identifizierung von molekularen Targets in vivo im Primärtumor und in Metastasen, Targets, die eine frühzeitige Diagnose und die Evaluierung von Progress und Therapieresponse ermöglichen.

5. Etablierung von durch bildgebende Verfahren gesteuerte und für den individuellen Patienten maßgeschneiderten Therapien.

6. Entwicklung bildgebender Verfahren für das In-vivo-Screening von Medikamenten mit Hilfe von Tiermodellen, mit denen sich für den Menschen relevante Vorhersagen treffen lassen.

7. Molekulare Bildgebung der Apoptose in malignen Tumoren.

Das EMIL-Netz ist in drei Ebenen organisiert:

Level-1-Partner fungieren als koordinierende und administrative Zentren für verschiedene Aktivitäten. Insgesamt gibt es neun Level-1-Partner.
Level-2-Partner sind Gruppen von europäischen Labors, die direkt aus dem EMIL-Budget finanziert werden. Die Tätigkeiten jeder Level-2-Partner-Gruppe werden von einem Level-1-Partner koordiniert. Derzeit gibt es insgesamt 40 Level-2-Partner.

Level-3-Partner sind europäische Labors, die ebenfalls als Gruppen von einem Level-1-Partner koordiniert werden. Sie tragen mit ihrem Know-how und ihren Techniken zu den EMIL-Aktivitäten bei, werden aber nicht direkt aus dem EMILBudget finanziert. Derzeit gibt es 13 Level3-Partner.

Das urologische Forschungslabor (Partner 48, Level 2, Klinik und Poliklinik für Urologie der Universität Bern, Schweiz) ist involviert in das Work Package (WP) 2: „Entwicklung von Genkonstruktionen, Zelllinien und neuen Krebsmodellen für die Biolumineszenz (BLI)und multimodale Bildgebung“.

Dank rascher Fortschritte im Bereich molekularbiologischer Instrumente und dank neuer Entwicklungen von extrem sensiblen Photonendetektoren können FLI („fluorescent imaging“, Fluoreszenz-Bildgebung) und BLI („bioluminescent imaging “, Biolumineszenz-Bildgebung) inzwischen nicht mehr nur zur Untersuchung zell- und gewebespezifische Promotoren verwendet werden, sondern auch dazu, die Bewegungen und das Schicksal der Zellen $\mathrm{zu}$ verfolgen, die grün fluoreszierendes Protein und/oder Luciferase exprimieren, sowie Apoptose, Protein-Protein-Interaktionen und Gentransfer. In der Krebsforschung haben FLI und BLI quantitative Messungen von Tumorprogress, Metastasenlast und Therapierespons ermöglicht. Aufgrund ihrer hohen Sensitivität sind FLI und besonders BLI außerordentlich hilfreich bei der Detektion von Mikrometastasen und minimaler Resterkrankung („minimal residual disease“, MRD) in Tiermodellen. Die Anwendung dieser innovativen Techniken ist allerdings noch auf Tiermodelle beschränkt, auch wenn sich die meisten möglicherweise für klinische Zwecke weiterentwickeln lassen.

In enger Kooperation mit Clemens W.G.M. Löwik (Direktor des Labors für Endokrinologische Forschung und der Abteilung für Molekulare Bildgebung, Departments of Endocrinology and Metabo- 
lic Diseases, Leiden University Medical Center, Leiden, Niederlande, Partner 4, Level 1) ist das Departement für Urologie der Universität Bern beteiligt an der Entwicklung eines spontanen Mausmodells für Prostatakrebs, in dem das Monitoring sowohl der Entwicklung des Primärtumors als auch der distanten Metastasierung über BLI verfolgt werden kann. Hierbei werden neuartige multimodale chimäre Reporterkonstrukte entwickelt, mit denen sich Tumor-Zelllinien generieren lassen, die eben diese Konstrukte stabil exprimieren und die geeignet sind für verschiedene bildgebende Techniken bzw. für Kombinationen dieser Techniken. Darüber hinaus werden wir auch neue In-vivo-Tiermodelle zur Darstellung der spontanen Tumorentwicklung, des Progresses und der Metastasierung entwickeln und Anstrengungen unternehmen, die optische Bildgebung mit anderen nichtinvasiven bildgebenden Modalitäten zu integrieren.

Diese neuen Methoden und Modellsysteme werden es uns ermöglichen, die Wirksamkeit neuer therapeutischer Ansätze wie beispielsweise Gentherapie oder Antiangiogenese zu überprüfen - bei Erfolg wird dies möglicherweise ein erster Schritt in Richtung klinischer Anwendung sein.

EMIL wird sich dauerhaft und langfristig in die Europäische Forschungslandschaft integrieren - dank akademischer Exzellenz, starker und stabiler Zusammenarbeit mit kleinen und mittelständischen Unternehmen („small to medium-sized enterprises“, SMEs), dank seiner Ausbildungsaktivitäten, des wissenschaftlichen Managements und eines Forschungsprogramms, das auf bereits Bestehendem basiert und anspruchsvolle Ziele verfolgt: Auf diese Weise wird die kritische Masse erreicht, die für eine internationale Führungsrolle und zur Überbrückung von Lücken im Innovationssystem erforderlich ist.

\section{Korrespondenzadresse \\ Prof. Dr. G. Thalmann \\ Urologische Universitätsklinik, \\ Anna Seiler-Haus \\ Inselspital, \\ 3010 Bern, Schweiz \\ George.thalmann@insel.ch}

Interessenkonflikt. Der korrespondierende Autor gibt an, dass kein Interessenkonflikt besteht.

Urologe 2007 · 46:1278-1285 · DOI 10.1007/s00120-007-1396-4 · Online publiziert: 14. Juli 2007

(c) Springer Medizin Verlag 2007

\author{
A. Heidenreich · C.H. Ohlmann · E. Özgür · D. Pfister - D. Sahi · D. Thüer · \\ U.H. Engelmann \\ Klinik und Poliklinik für Urologie, Uniklinik, Köln
}

\title{
Behandlungsoptimierung fortgeschrittener urologischer Malignome
}

\begin{abstract}
Neben den Aufgaben einer qualitativ hochwertigen Patientenversorgung unter Berücksichtigung innovativer und experimenteller Therapieverfahren stellen die Bereiche der Lehre und Forschung weitere Eckpfeiler der universitären Medizin dar. Zielsetzung der akademisch orientierten Urologie sollte es sein, einen Transferprozess zwischen der auf die Ergründung der molekularen, pathogenetischen und pathophysiologischen Ursache verschiedener krankhafter oder pathologisch alterierter Stoffwechselprozesse ausgerichteten Grundlagenforschung und der auf die unmittelbare Behandlung bedeutsamer Krankheitsbilder ausgerichteten klinischen Forschung zu realisieren.
\end{abstract}

Nur durch die intensive Diskussion zwischen Klinikern und Grundlagenwissenschaftlern wird es möglich sein, die Therapie insbesondere chronischer oder unheilbarer Erkrankungen im Sinne der Patienten zu optimieren. Unabdingbare Voraussetzung für eine derartige, für Patient und Mediziner fruchtbare Diskussion ist eine Schwerpunktbildung der Forschungsaktivitäten innerhalb einer akademischen Einrichtung des Fachgebiets der Urologie. Die Schwerpunktbildung erlaubt zum einen die breite wissenschaftliche Ausrichtung einer urologischen Universitätsklinik, zum anderen eine qualitativ hochwertige Wissenschaft durch die Besetzung der verschiedenen Forschungsfelder mit in den wissenschaftlichen und klinischen Belangen des Forschungsbereichs ausgewiesenen Experten. Die jeweiligen Be- reichsleiter sind für die Qualität, Standardisierung und Modernisierung der Diagnostik und Therapie der jeweiligen Schwerpunkte ebenso verantwortlich wie für eine fundierte klinische und wissenschaftliche Ausbildung der ärztlichen Mitarbeiterinnen und Mitarbeiter. Zudem ist es Aufgabe der Bereichsleiter, durch eine interdisziplinäre, horizontale und vertikale Vernetzung zwischen Klinik und universitätszugehörigen Institutionen der Grundlagenforschung ein funktionierendes wissenschaftliches Netzwerk aufzubauen (• Abb. 1).

Im Folgenden stellen wir die aktuellen Ergebnisse des Schwerpunktes Urologische Onkologie der Klinik und Poliklinik für Urologie der Universität zu Köln dar, die sich auf die Behandlungsoptimierung von Patienten mit fortgeschrittenem testikulären Keimzelltumor, Urothelkarzinom der Harnblase und des oberen Harntrakts sowie des androgenunabhängigen Prostatakarzinoms konzentrieren.

\section{Urologische Onkologie}

\section{Behandlungsoptimierung und Grundlagenforschung beim testi- kulären Keimzelltumor}

Die Residualtumorresektion (RTR) nach systemischer Chemotherapie stellt ein Standardvorgehen in dem multimodalen Therapiekonzept fortgeschrittener nichtseminomatöser testikulärer Keimzelltumoren (NSKZT) dar, nachdem ca. $35 \%$ bzw. 15\% der Patienten mit einem NSKZT matures Teratom oder vitales Karzinom in den Residuen aufweisen [12, 27]. Es ist 